\title{
ON THE RIESZ TRANSFORMS FOR THE INVERSE GAUSS MEASURE
}

\author{
Tommaso Bruno and Peter Sjögren \\ Politecnico di Torino, Dipartimento di Scienze Matematiche "Giuseppe Luigi Lagrange" \\ Corso Duca degli Abruzzi 24, 10129 Torino, Italy; and \\ Ghent University, Department of Mathematics: Analysis, Logic and Discrete Mathematics \\ Krijgslaan 281, 9000 Ghent, Belgium; tommaso.bruno@ugent.be \\ Chalmers University of Technology, Mathematical Sciences; and University of Gothenburg, \\ Mathematical Sciences, SE-41296 Göteborg, Sweden; peters@chalmers.se
}

\begin{abstract}
Let $\gamma_{-1}$ be the absolutely continuous measure on $\mathbf{R}^{n}$ whose density is the reciprocal of a Gaussian function. Let further $\mathscr{A}$ be the natural self-adjoint Laplacian on $L^{2}\left(\gamma_{-1}\right)$. In this paper, we prove that the Riesz transforms associated with $\mathscr{A}$ of order one or two are of weak type $(1,1)$, but that those of higher order are not.
\end{abstract}

\section{Introduction}

The Euclidean space endowed with the measure $\gamma_{-1}$ whose density is the reciprocal of a Gaussian, which we call the inverse Gauss measure, is a toy model of a variety of settings where a theory of singular integral operators has not yet been established. Therefore, the analysis of this model may provide profitable insights into more general frameworks. In this paper, we focus on the boundedness of the Riesz transforms.

As a weighted manifold, $\left(\mathbf{R}^{n}, \gamma_{-1}\right)$ has constant, negative definite Bakry-Émery curvature tensor. By a celebrated result of Bakry on weighted Riemannian manifolds [2], the negative lower bound of this tensor governs the $L^{p}$ boundedness, $1<p<\infty$, of the shifted Riesz transforms associated with the natural weighted Laplacian on the manifold. No general endpoint analogue is known. In addition to this, the natural weighted Laplacian $\mathscr{A}$ on $\left(\mathbf{R}^{n}, \gamma_{-1}\right)$ can be seen as a restriction of the Laplace-Beltrami operator on a warped-product manifold whose Ricci tensor is unbounded from below. Not much is known about Riesz transforms on manifolds of unbounded geometry; see for instance $[1,5]$ and references therein. We also emphasize that the inverse Gauss measure setting is intimately related to the Gaussian setting, for $\mathscr{A}$ is unitarily equivalent with a translate of the Ornstein-Uhlenbeck operator.

The connection with the Gaussian setting was the principal motivation for which $\gamma_{-1}$ and $\mathscr{A}$ were initially introduced and studied by Salogni [9]. Then, several endpoint results for imaginary powers and Riesz transforms of $\mathscr{A}$, involving also new spaces of Hardy type, were obtained in [4] by the first author of the present paper. There, it was proved that if $\lambda \geq 1$, then the shifted first-order Riesz transforms $\nabla(\mathscr{A}+\lambda I)^{-1 / 2}$ are of weak type $(1,1)$, that is, bounded from $L^{1}\left(\gamma_{-1}\right)$ to $L^{1, \infty}\left(\gamma_{-1}\right)$.

https://doi.org/10.5186/aasfm.2021.4609

2020 Mathematics Subject Classification: Primary 42B20, 47B03.

Key words: Inverse Gauss measure, Riesz transforms, weak type $(1,1)$.

T. Bruno was partially supported by the Research Foundation - Flanders (FWO) through the postdoctoral grant 12ZW120N. P. Sjögren is grateful to the Università di Genova and the Politecnico di Torino for supporting visits which made this work possible. 
This result, and those concerning Hardy spaces, strongly resemble endpoint analogues of the theorem of Bakry mentioned above. However, the problem whether such a shift is indeed necessary was left open.

The aim of this paper is to study the weak type $(1,1)$ of the Riesz transforms of any order of $\mathscr{A}$. In particular, we improve the first-order results by eliminating the shift. This was unexpected, because the shift is necessary for the boundedness from Hardy spaces adapted to $\gamma_{-1}$ to $L^{1}\left(\gamma_{-1}\right)$, as proved in [4]. Another surprising fact is that the weak type $(1,1)$ behaviour of the Riesz transforms of $\mathscr{A}$ is completely analogous to that of the Riesz transforms of the Ornstein-Uhlenbeck operator, although the behaviour on corresponding Hardy spaces is different. See [6, 8] and [4, 3], respectively.

To be more explicit, let $\alpha=\left(\alpha_{1}, \ldots, \alpha_{n}\right) \in \mathbf{N}^{n}$ be a nonzero multi-index and let $\mathscr{R}_{\alpha}=\partial^{\alpha} \mathscr{A}^{-|\alpha| / 2}$, which is a Riesz transform of order $|\alpha|=\alpha_{1}+\cdots+\alpha_{n}$. In this paper, we prove the following.

Theorem 1.1. Let $\alpha \in \mathbf{N}^{n}$ be nonzero. Then $\mathscr{R}_{\alpha}$ is bounded from $L^{1}\left(\gamma_{-1}\right)$ to $L^{1, \infty}\left(\gamma_{-1}\right)$ if and only if $|\alpha| \leq 2$.

By [4, Remark 2.5], $\mathscr{R}_{\alpha}$ is bounded on $L^{p}\left(\gamma_{-1}\right)$ for every $p \in(1, \infty)$ when $|\alpha|=1$. We do not know whether the same holds in general when $|\alpha|>1$, though we strongly expect so; we leave the investigation of this problem to future work.

The paper is devoted to the proof of Theorem 1.1. We determine the integral kernel of the Riesz transform $\mathscr{R}_{\alpha}$ and split it into a local and a global part. The local part behaves like a classical Calderón-Zygmund kernel, and its weak type $(1,1)$ follows by an adaptation of the classical Calderón-Zygmund theory, developed in [9]. This boundedness holds for the Riesz transforms of any order. The heart of the proof concerns the global part of the kernel, which requires ad hoc techniques where the order of the transforms matters.

The paper is organized as follows. In Section 2, the operator $\mathscr{A}$, its Mehler-type kernel and its Riesz transforms are defined. We then prove the weak type $(1,1)$ of the local parts of the Riesz transforms in Section 3. In Section 4, we prove weak type $(1,1)$ estimates for operators with several kinds of kernels. These estimates will allow us, in Section 5, to complete the proof of the "if" part of Theorem 1.1. In the final Section 6 , it is shown that the weak type $(1,1)$ of the Riesz transforms of order higher than two cannot hold.

We now explain some notation. Let $\underline{0}$ denote the null vector $(0, \ldots, 0) \in \mathbf{N}^{n}$. For $\alpha \in \mathbf{N}^{n} \backslash\{\underline{0}\}$, we write $\partial^{\alpha}$ for the differential operator $\partial_{x_{1}}^{\alpha_{1}} \cdots \partial_{x_{n}}^{\alpha_{n}}$. The Lebesgue measure on $\mathbf{R}^{n}$ will be denoted by $d x$ or by Leb. If $E$ is a measurable set, $\mathbf{1}_{E}$ will stand for its characteristic function. Given a linear operator $T$ mapping test functions into measurable functions on $\mathbf{R}^{n}$, we say that a measurable function $K$ defined and locally bounded off the diagonal in $\mathbf{R}^{n} \times \mathbf{R}^{n}$ is the integral kernel of $T$ if, for any test function $f$,

$$
T f(x)=\int_{\mathbf{R}^{n}} K(x, y) f(y) d y, \quad x \notin \operatorname{supp}(f) .
$$

In other words, the integral kernels of our operators will be taken with respect to Lebesgue measure.

We denote by $C<\infty$, or $c>0$, a constant that may vary from place to place but is independent of significant quantities. Given two positive quantities $A$ and $B$, we shall write $A \lesssim B$ or $B \gtrsim A$ if $A \leq C B$. If $A \lesssim B$ and $B \lesssim A$, we write $A \approx B$. The symbols $A \vee B$ and $A \wedge B$ will stand for $\max (A, B)$ and $\min (A, B)$ respectively. 


\section{The operator $\mathscr{A}$ and its Riesz transforms}

For $x \in \mathbf{R}^{n}$, let $\gamma(x)=\pi^{-n / 2} e^{-|x|^{2}}$ and $\gamma_{-1}(x)=\pi^{n / 2} e^{|x|^{2}}$. With a slight abuse of notation, we identify $\gamma$ and $\gamma_{-1}$ with the measures $\gamma(x) d x$ and $\gamma_{-1}(x) d x$, respectively. Consider the second order differential operator

$$
\mathscr{A}_{0} f(x)=-\frac{1}{2} \Delta f(x)-x \cdot \nabla f(x), \quad f \in C_{c}^{\infty}\left(\mathbf{R}^{n}\right), x \in \mathbf{R}^{n},
$$

which is essentially self-adjoint on $L^{2}\left(\gamma_{-1}\right)$. Denote with $\mathscr{A}$ its closure. It is known, see [9] and [4], that the $L^{2}\left(\gamma_{-1}\right)$-spectrum of $\mathscr{A}$ is the discrete set $\{n, n+1, \ldots\}$, and that its eigenfunctions are the functions $\left(\gamma H_{\alpha}\right)_{\alpha \in \mathbf{N}^{n}}$, where $H_{\alpha}$ is an $n$-dimensional Hermite polynomial, i.e.,

$$
H_{\alpha}=\bigotimes_{j=1}^{n} H_{\alpha_{j}}, \quad H_{k}(s)=(-1)^{k} e^{s^{2}} \frac{d^{k}}{d s^{k}} e^{-s^{2}}, \quad k \in \mathbf{N}, s \in \mathbf{R} .
$$

Recall that for $t>0$, the integral kernel of the operator $e^{-t \mathscr{A}}$ is

$$
H_{t}(x, y)=\frac{e^{-n t}}{\pi^{n / 2}\left(1-e^{-2 t}\right)^{n / 2}} e^{-\frac{\left|x-e^{-t} y\right|^{2}}{1-e^{-2 t}}}, \quad x, y \in \mathbf{R}^{n}
$$

see [9] and [4] for this and further details about $\mathscr{A}$.

For $b>0$, the kernel of $\mathscr{A}^{-b}$ is given by the subordination formula

$$
K_{\mathscr{A}^{-b}}(x, y)=\Gamma(b)^{-1} \int_{0}^{\infty} t^{b-1} H_{t}(x, y) d t
$$

The kernel of the Riesz transform $\mathscr{R}_{\alpha}=\partial^{\alpha} \mathscr{A}^{-|\alpha| / 2}$ with $\alpha \in \mathbf{N}^{n} \backslash\{\underline{0}\}$ is

$$
K_{\mathscr{R}_{\alpha}}(x, y)=\pi^{-n / 2} \Gamma(|\alpha| / 2)^{-1} \int_{0}^{\infty} t^{\frac{|\alpha|}{2}-1} \frac{e^{-n t}}{\left(1-e^{-2 t}\right)^{n / 2}} \partial_{x}^{\alpha} e^{-\frac{\left|x-e^{-t} y\right|^{2}}{1-e^{-2 t}}} d t
$$

in the principal value sense. By the change of variables $t=-\log r$, and recalling (2.1), we obtain that the kernel of $\mathscr{R}_{\alpha}$ is the principal value of

$$
\begin{aligned}
K_{\mathscr{R}_{\alpha}}(x, y) & =\frac{1}{\pi^{n / 2} \Gamma(|\alpha| / 2)} \int_{0}^{1} \frac{r^{n-1}(-\log r)^{\frac{|\alpha|}{2}-1}}{\left(1-r^{2}\right)^{n / 2}} \partial_{x}^{\alpha} e^{-\frac{|x-r y|^{2}}{1-r^{2}}} d r \\
(2.2) & =\frac{(-1)^{|\alpha|}}{\pi^{n / 2} \Gamma(|\alpha| / 2)} \int_{0}^{1} \frac{r^{n-1}(-\log r)^{\frac{|\alpha|}{2}}-1}{\left(1-r^{2}\right)^{(n+|\alpha|) / 2}} H_{\alpha}\left(\frac{x-r y}{\sqrt{1-r^{2}}}\right) e^{-\frac{|x-r y|^{2}}{1-r^{2}}} d r \\
(2.3) & =\frac{(-1)^{|\alpha|}}{\pi^{n / 2} \Gamma(|\alpha| / 2)} e^{-|x|^{2}+|y|^{2}} \int_{0}^{1} \frac{r^{n-1}(-\log r)^{\frac{|\alpha|}{2}-1}}{\left(1-r^{2}\right)^{(n+|\alpha|) / 2}} H_{\alpha}\left(\frac{x-r y}{\sqrt{1-r^{2}}}\right) e^{-\frac{|r x-y|^{2}}{1-r^{2}}} d r,
\end{aligned}
$$

the last equality since

$$
-\frac{|x-r y|^{2}}{1-r^{2}}+\frac{|r x-y|^{2}}{1-r^{2}}=-|x|^{2}+|y|^{2} .
$$

We split $\mathbf{R}^{n} \times \mathbf{R}^{n}$ into a local and a global region. For $\delta=1,2$, define

$$
N_{\delta}=\left\{(x, y) \in \mathbf{R}^{n} \times \mathbf{R}^{n}:|x-y| \leq \frac{\delta}{1+|x|+|y|}\right\}
$$

and $G=N_{1}^{c}$. The regions $N_{1}$ and $N_{2}$ will be called local regions, while $G$ will be the global region. As in [4], we fix a smooth function $\chi: \mathbf{R}^{n} \times \mathbf{R}^{n} \rightarrow \mathbf{R}$ such that

$$
\mathbf{1}_{N_{1}} \leq \chi \leq \mathbf{1}_{N_{2}}, \quad\left|\nabla_{x} \chi(x, y)\right|+\left|\nabla_{y} \chi(x, y)\right| \leq \frac{C}{|x-y|} \quad \text { for every } x \neq y .
$$


We also define

$$
K_{\mathscr{R}_{\alpha}, \text { loc }}=\chi K_{\mathscr{R}_{\alpha}}, \quad K_{\mathscr{R}_{\alpha}, \text { glob }}=K_{\mathscr{R}_{\alpha}}-K_{\mathscr{R}_{\alpha}, \text { loc }}
$$

We shall denote the operators with kernels (the principal value of) $K_{\mathscr{R}_{\alpha} \text {,loc }}$ and $K_{\mathscr{R}_{\alpha} \text {,glob }}$ by $\mathscr{R}_{\alpha, \text { loc }}$ and $\mathscr{R}_{\alpha, \text { glob }}$, respectively. Theorem 1.1 will be proved by putting together Proposition 3.1 (boundedness of $\mathscr{R}_{\alpha, \text { loc }}$ for every $\alpha$ ), Proposition 5.1 (boundedness of $\mathscr{R}_{\alpha, \text { glob }}$ when $|\alpha| \leq 2$ ), and Proposition 6.1 (unboundedness of $\mathscr{R}_{\alpha}$ when $|\alpha| \geq 3)$.

\section{The local part of the Riesz transforms}

In this section, we prove the boundedness of $\mathscr{R}_{\alpha, \text { loc }}$. As we shall see, no restriction on $\alpha$ is necessary: indeed, in the local region all the Riesz transforms of $\mathscr{A}$ behave essentially like classical Calderón-Zygmund operators.

Proposition 3.1. $\mathscr{R}_{\alpha, \text { loc }}$ is bounded from $L^{1}\left(\gamma_{-1}\right)$ to $L^{1, \infty}\left(\gamma_{-1}\right)$ for every $\alpha \in$ $\mathbf{N}^{n} \backslash\{\underline{0}\}$.

The proof of Proposition 3.1 will be reduced to proving the boundedness of $\mathscr{R}_{\alpha}$ on $L^{2}\left(\gamma_{-1}\right)$ and some Calderón-Zygmund type estimates of $K_{\mathscr{R}_{\alpha}, \text { loc }}$. Following [9], we say that a linear operator $T$, mapping test functions into measurable functions on $\mathbf{R}^{n}$, is a local Calderón-Zygmund operator if

(a) $T$ is a bounded operator on $L^{q}\left(\gamma_{-1}\right)$ for some $q \in(1, \infty)$;

(b) $T$ has an integral kernel $K$, defined and locally integrable off the diagonal in $\mathbf{R}^{n} \times \mathbf{R}^{n}$, satisfying

$$
|K(x, y)| \lesssim \frac{1}{|x-y|^{n}}, \quad\left|\nabla_{x} K(x, y)\right|+\left|\nabla_{y} K(x, y)\right| \lesssim \frac{1}{|x-y|^{n+1}}
$$

for all $(x, y) \in N_{2}, x \neq y$.

If $\mathscr{R}_{\alpha}$ is a local Calderón-Zygmund operator, then $\mathscr{R}_{\alpha, \text { loc }}$ is bounded on $L^{p}\left(\gamma_{-1}\right)$ for any $p \in(1, \infty)$, and from $L^{1}\left(\gamma_{-1}\right)$ to $L^{1, \infty}\left(\gamma_{-1}\right)$; see e.g. [9, Theorem 3.2.8].

When $|\alpha|=1$, the boundedness of $\mathscr{R}_{\alpha, \text { loc }}$ was proved in [4, Proposition 6.6]. We now extend that argument to any nonzero $\alpha$. The key tool will be the following lemma, see [4, Lemma 6.3].

Lemma 3.2. Let $\mu, \nu \geq 0$ be such that $\mu>\nu+1$. Then, for every $(x, y) \in N_{2}$, $x \neq y$,

$$
\int_{0}^{1} \frac{|x-r y|^{\nu}}{\left(1-r^{2}\right)^{\frac{n+\mu}{2}}} e^{-\frac{|x-r y|^{2}}{1-r^{2}}} d r \lesssim \frac{1}{|x-y|^{n+\mu-\nu-2}} .
$$

We are now in a position to prove Proposition 3.1.

Proof of Proposition 3.1. It is enough to prove that $\mathscr{R}_{\alpha}$ is a local CalderónZygmund operator.

Step 1. We prove that $K_{\mathscr{R}_{\alpha}}$ satisfies the estimates (3.1). First, observe that by (2.2)

$$
\begin{aligned}
K_{\mathscr{R}_{\alpha}}(x, y) \lesssim & \int_{0}^{1 / 2} r^{n-1}(-\log r)^{\frac{|\alpha|}{2}-1}\left|H_{\alpha}\left(\frac{x-r y}{\sqrt{1-r^{2}}}\right)\right| e^{-\frac{|x-r y|^{2}}{1-r^{2}}} d r \\
& +\int_{1 / 2}^{1} \frac{1}{(1-r)^{\frac{n}{2}+1}}\left|H_{\alpha}\left(\frac{x-r y}{\sqrt{1-r^{2}}}\right)\right| e^{-\frac{|x-r y|^{2}}{1-r^{2}}} d r .
\end{aligned}
$$


Since

$$
\left|H_{\alpha}\left(\frac{x-r y}{\sqrt{1-r^{2}}}\right)\right| \lesssim \sum_{a=0}^{|\alpha|}\left(\frac{|x-r y|}{\sqrt{1-r^{2}}}\right)^{a},
$$

and $s^{a} e^{-s^{2}} \lesssim 1$ for every $a \geq 0$ with a constant depending only on $a$,

$$
\int_{0}^{1 / 2} r^{n-1}(-\log r)^{\frac{|\alpha|}{2}-1}\left|H_{\alpha}\left(\frac{x-r y}{\sqrt{1-r^{2}}}\right)\right| e^{-\frac{|x-r y|^{2}}{1-r^{2}}} d r \lesssim \int_{0}^{1 / 2} r^{n-1}(-\log r)^{\frac{|\alpha|}{2}-1} d r \lesssim 1 .
$$

Moreover, by Lemma 3.2 we obtain

$$
\begin{aligned}
\int_{1 / 2}^{1} \frac{1}{(1-r)^{\frac{n}{2}+1}}\left|H_{\alpha}\left(\frac{x-r y}{\sqrt{1-r^{2}}}\right)\right| e^{-\frac{|x-r y|^{2}}{1-r^{2}}} d r & \lesssim \sum_{a=0}^{|\alpha|} \int_{0}^{1} \frac{|x-r y|^{a}}{\left(1-r^{2}\right)^{\frac{n+2+a}{2}}} e^{-\frac{|x-r y|^{2}}{1-r^{2}}} d r \\
& \lesssim \frac{1}{|x-y|^{n}}
\end{aligned}
$$

The estimates of the gradients of $K_{\mathscr{R}_{\alpha}}$ can be obtained analogously. Indeed,

$$
\left|\partial_{x_{j}}\left[H_{\alpha}\left(\frac{x-r y}{\sqrt{1-r^{2}}}\right) e^{-\frac{|x-r y|^{2}}{1-r^{2}}}\right]\right| \lesssim e^{-\frac{|x-r y|^{2}}{1-r^{2}}}\left(\sum_{a=1}^{|\alpha|} \frac{|x-r y|^{a-1}}{\left(1-r^{2}\right)^{\frac{a}{2}}}+\sum_{a=0}^{|\alpha|} \frac{|x-r y|^{a+1}}{\left(1-r^{2}\right)^{\frac{a}{2}+1}}\right),
$$

and the same estimates hold for the derivative along $y_{j}$. The integral over $(0,1 / 2)$ goes as before, and by Lemma 3.2

$$
\begin{aligned}
& \left|\nabla_{x} K_{\mathscr{R}_{\alpha}}(x, y)\right|+\left|\nabla_{y} K_{\mathscr{R}_{\alpha}}(x, y)\right| \\
& \lesssim 1+\left(\sum_{a=1}^{|\alpha|} \int_{1 / 2}^{1} \frac{|x-r y|^{a-1}}{\left(1-r^{2}\right)^{\frac{n+2+a}{2}}} e^{-\frac{|x-r y|^{2}}{1-r^{2}}} d r+\sum_{a=0}^{|\alpha|} \int_{1 / 2}^{1} \frac{|x-r y|^{a+1}}{\left(1-r^{2}\right)^{\frac{n+4+a}{2}}} e^{-\frac{|x-r y|^{2}}{1-r^{2}}} d r\right) \\
& \lesssim \frac{1}{|x-y|^{n+1}} .
\end{aligned}
$$

Thus, the estimates (3.1) hold for $K_{\mathscr{R}_{\alpha}}$.

Step 2. We prove that $\mathscr{R}_{\alpha}$ is bounded on $L^{2}\left(\gamma_{-1}\right)$. For $\beta \in \mathbf{N}^{n}$, we denote by $h_{\beta}$ the normalized Hermite polynomial

$$
h_{\beta}=2^{-\frac{|\beta|}{2}}(\beta !)^{-1 / 2} H_{\beta} .
$$

The functions $\gamma h_{\beta}$ form an orthonormal basis in $L^{2}\left(\gamma_{-1}\right)$, see e.g. [11, §5.5]. Since for $b>0$

$$
\mathscr{A}^{-b} f=\sum_{\beta \in \mathbf{N}^{n}} \frac{1}{(|\beta|+n)^{b}}\left(f, \gamma h_{\beta}\right)_{L^{2}(\gamma-1)} \gamma h_{\beta},
$$

and, by the definition of $h_{\beta}$ and $H_{\beta}$,

$$
\partial^{\alpha}\left(\gamma h_{\beta}\right)=2^{\frac{|\alpha|}{2}}(-1)^{|\alpha|+|\beta|} \sqrt{\frac{(\beta+\alpha) !}{\beta !}} \gamma h_{\beta+\alpha}
$$

we obtain

$$
\begin{aligned}
\mathscr{R}_{\alpha} f & =\partial^{\alpha} \mathscr{A}^{-|\alpha| / 2} f=\sum_{\beta} \frac{1}{(|\beta|+n)^{|\alpha| / 2}}\left(f, \gamma h_{\beta}\right)_{L^{2}\left(\gamma_{-1}\right)} \partial^{\alpha}\left(\gamma h_{\beta}\right) \\
& =2^{|\alpha| / 2} \sum_{\beta}(-1)^{|\alpha|+|\beta|} \frac{1}{(|\beta|+n)^{|\alpha| / 2}}\left(f, \gamma h_{\beta}\right)_{L^{2}(\gamma-1)} \sqrt{\frac{(\beta+\alpha) !}{\beta !}} \gamma h_{\beta+\alpha} .
\end{aligned}
$$


Since the $\left(\gamma h_{\nu}\right)$ are orthonormal in $L^{2}\left(\gamma_{-1}\right)$, and

$$
\frac{1}{(|\beta|+n)^{|\alpha|}} \frac{(\beta+\alpha) !}{\beta !} \lesssim 1
$$

where the implicit constant may depend on $\alpha$ and $n$, we conclude

$$
\begin{aligned}
\left\|\mathscr{R}_{\alpha} f\right\|_{L^{2}(\gamma-1)}^{2} & \left.=2^{|\alpha|} \sum_{\beta} \frac{1}{(|\beta|+n)^{|\alpha|}} \frac{(\beta+\alpha) !}{\beta !}\left(f, \gamma h_{\beta}\right)_{L^{2}(\gamma-1}^{2}\right) \\
& \lesssim 2^{|\alpha|} \sum_{\beta}\left(f, \gamma h_{\beta}\right)_{L^{2}(\gamma-1)}^{2} \lesssim\|f\|_{L^{2}(\gamma-1)}^{2} .
\end{aligned}
$$

This concludes Step 2 and proves that $\mathscr{R}_{\alpha}$ is a local Calderón-Zygmund operator. The proposition follows.

\section{Some technical lemmata}

In this section, we prove some lemmata concerning the weak type $(1,1)$ of various integral operators with given kernels. The kernels are those appearing in the analysis of the Riesz transforms of order 1 and 2 in the next section.

We first introduce some notation, which will be used in the rest of the paper. Given a couple $(x, y) \in \mathbf{R}^{n} \times \mathbf{R}^{n}, x \neq \underline{0}$, we shall write

$$
y=y_{x}+y_{\perp},
$$

where $y_{x}$ is parallel to $x$ and $y_{\perp}$ is orthogonal to $x$. We denote with $r_{0}$ the unique real number such that $y_{x}=r_{0} x$, and let $\theta=\theta(x, y) \in[0, \pi]$ be the angle between $x$ and $y$. Then,

$$
r_{0}=(|y| /|x|) \cos \theta, \quad\left|r_{0}\right|=\left|y_{x}\right| /|x|, \quad\left|y_{\perp}\right|=|y| \sin \theta .
$$

Observe moreover that

$$
|r x-y|^{2}=\left|r-r_{0}\right|^{2}|x|^{2}+\left|y_{\perp}\right|^{2}
$$

that

$$
\left|1-r_{0}\right|=\left|x-y_{x}\right| /|x|
$$

and that

$$
|x-r y| \leq\left|x-y_{x}\right|+\left|y_{x}-r y\right| \leq\left|x-y_{x}\right|+(1-r)\left|y_{x}\right|+r\left|y_{\perp}\right| .
$$

Notice also that $|x \pm y| \geq|x| \sin \theta$ and that, if $(x, y) \in G$, then $|x-y| \geq \frac{1}{2}(1+|x|)^{-1}$, see e.g. [4, Lemma 6.2].

It will be useful to recall that if $T$ is a linear operator such that

$$
T=\sum_{m=0}^{\infty} T_{m}
$$

for some linear operators $\left(T_{m}\right)_{m \in \mathbf{N}}$, each bounded from $L^{1}\left(\gamma_{-1}\right)$ to $L^{1, \infty}\left(\gamma_{-1}\right)$, then

$$
\begin{aligned}
& \|T\|_{L^{1}\left(\gamma_{-1}\right) \rightarrow L^{1, \infty}\left(\gamma_{-1}\right)} \\
& \leq \sum_{m=0}^{\infty}\left\|T_{m}\right\|_{L^{1}\left(\gamma_{-1}\right) \rightarrow L^{1, \infty}\left(\gamma_{-1}\right)}\left(1+\log \left(1+\left\|T_{m}\right\|_{L^{1}\left(\gamma_{-1}\right) \rightarrow L^{1, \infty}\left(\gamma_{-1}\right)}\right)\right),
\end{aligned}
$$

see [10, Lemma 2.3]. 
We now begin with the lemmata giving the weak type $(1,1)$ of some integral operators. The first is nothing but [9, Lemma 3.3.4] (see also [6, Theorem 1]), which we restate for the reader's convenience.

Lemma 4.1. The operator with kernel

$$
K(x, y)=e^{-|x|^{2}+|y|^{2}}\left[(1+|x|)^{n} \wedge(|x| \sin \theta)^{-n}\right]
$$

is bounded from $L^{1}\left(\gamma_{-1}\right)$ to $L^{1, \infty}\left(\gamma_{-1}\right)$.

Lemma 4.2. Let $\mu, \nu \in \mathbf{R}$ be such that $\mu+\nu \geq n-2$ and $\mu \leq n$. Then the operator $T$ with kernel

$$
K(x, y)=\pi^{n / 2} e^{-|x|^{2}+|y|^{2}}|x|^{-\mu}(1+|x|)^{-\nu}
$$

is bounded from $L^{1}\left(\gamma_{-1}\right)$ to $L^{1, \infty}\left(\gamma_{-1}\right)$.

Proof. Let $f \in L^{1}\left(\gamma_{-1}\right), f \geq 0$, and observe that

$$
T f(x)=e^{-|x|^{2}}|x|^{-\mu}(1+|x|)^{-\nu}\|f\|_{L^{1}\left(\gamma_{-1}\right)} .
$$

Now, consider for $s>0$ the set

$$
A_{s}=\{x: T f(x)>s\}=\left\{x: e^{-|x|^{2}}|x|^{-\mu}(1+|x|)^{-\nu}\|f\|_{L^{1}\left(\gamma_{-1}\right)}>s\right\} .
$$

Let $r=r_{s}$ be the largest positive solution of the equation

$$
e^{-r^{2}} r^{-\mu}(1+r)^{-\nu}\|f\|_{L^{1}(\gamma-1)}=s
$$

clearly such a solution exists unless $A_{s}$ is empty. Now $A_{s} \subset B\left(0, r_{s}\right)$ and if $r_{s} \leq 1$, then

$$
\gamma_{-1}\left(A_{s}\right) \leq \int_{B\left(0, r_{s}\right)} e^{|x|^{2}} d x \lesssim r_{s}^{n} \lesssim \frac{1}{s}\|f\|_{L^{1}\left(\gamma_{-1}\right)} r_{s}^{n-\mu} \lesssim \frac{1}{s}\|f\|_{L^{1}\left(\gamma_{-1}\right)}
$$

since $n-\mu \geq 0$. If instead $r_{s} \geq 1$,

$$
\gamma_{-1}\left(A_{s}\right) \leq \int_{B\left(0, r_{s}\right)} e^{|x|^{2}} d x \lesssim e^{r_{s}^{2}} r_{s}^{n-2} \lesssim \frac{1}{s}\|f\|_{L^{1}\left(\gamma_{-1}\right)} r_{s}^{n-\mu-\nu-2} \lesssim \frac{1}{s}\|f\|_{L^{1}\left(\gamma_{-1}\right)},
$$

since $n-\mu-\nu-2 \leq 0$. This completes the proof.

Lemma 4.3. Let $\delta>0$. Then, the operator $T$ with kernel

$$
K(x, y)=e^{-|x|^{2}+|y|^{2}} e^{-\delta\left|y_{\perp}\right|^{2}}|x|\left(\frac{|y|}{|x|}\right)^{n-1} \mathbf{1}_{G \cap\{|y| \leq 2|x|\}}(x, y)
$$

is bounded from $L^{1}\left(\gamma_{-1}\right)$ to $L^{1, \infty}\left(\gamma_{-1}\right)$.

Proof. Let $0 \leq f \in L^{1}\left(\gamma_{-1}\right)$. For $\underline{0} \neq x \in \mathbf{R}^{n}$, let $x^{\prime}=x /|x|$ be its projection on the unit sphere $\mathbf{S}^{n-1}$, which we endow with the standard normalized surface measure $d y^{\prime}$. Let

$$
\begin{aligned}
K_{0}(x, y) & =K(x, y) \mathbf{1}_{\left\{\left|y_{\perp}\right| \leq 1\right\}}(x, y), \\
K_{m}(x, y) & =K(x, y) \mathbf{1}_{\left\{2^{m-1}<\left|y_{\perp}\right| \leq 2^{m}\right\}}(x, y), \quad m=1,2, \ldots,
\end{aligned}
$$

so that

$$
K=\sum_{m=0}^{\infty} K_{m}
$$

and let $T_{m}$ be the operator whose kernel is $K_{m}$. Observe that if $\left|y_{\perp}\right| \leq 2^{m}$, then

$$
\left|y^{\prime}-x^{\prime}\right|=2 \sin (\theta / 2) \leq 2 \sin \theta \leq \frac{2^{m+1}}{|y|}
$$


when $\theta \in[0, \pi / 2]$, while if $\theta \in[\pi / 2, \pi]$, then

$$
\left|y^{\prime}+x^{\prime}\right|=2 \sin ((\pi-\theta) / 2) \leq 2 \sin \theta \leq \frac{2^{m+1}}{|y|} .
$$

Thus, with a constant $c=c(\delta)>0$

$$
\begin{aligned}
T_{m} f(x) & \lesssim|x|^{2-n} e^{-|x|^{2}} e^{-c 2^{2 m}} \int_{\left\{|y| \leq 2|x|,\left|y^{\prime}-x^{\prime}\right| \wedge\left|y^{\prime}+x^{\prime}\right| \leq 2^{m+1} /|y|\right\}}|y|^{n-1} f(y) e^{|y|^{2}} d y \\
& =|x|^{2-n} e^{-|x|^{2}} e^{-c 2^{2 m}} \int_{0}^{2|x|} \rho^{2 n-2}\left(\int_{\left\{\left|y^{\prime}-x^{\prime}\right| \wedge\left|y^{\prime}+x^{\prime}\right| \leq 2^{m+1} / \rho\right\}} f\left(\rho y^{\prime}\right) d y^{\prime}\right) e^{\rho^{2}} d \rho \\
& =|x|^{2-n} e^{-|x|^{2}} e^{-c 2^{2 m}} I_{m}\left(|x|, x^{\prime}\right),
\end{aligned}
$$

where the last equality defines $I_{m}\left(|x|, x^{\prime}\right)$. For $s>0$ let now $B_{s, m} \subset \mathbf{S}^{n-1}$ be the set of all $x^{\prime}$ such that the equation

$$
e^{-c 2^{2 m}} r^{2-n} e^{-r^{2}} I_{m}\left(r, x^{\prime}\right)=s
$$

admits a positive solution $r$; for $x^{\prime} \in B_{s, m}$, denote by $r_{s, m}\left(x^{\prime}\right)$ the largest solution, which exists since $I_{m}\left(r, x^{\prime}\right) \lesssim r^{n-1}\|f\|_{L^{1}\left(\gamma_{-1}\right)}$. Then

$$
A_{s, m}:=\left\{x: T_{m} f(x)>s\right\} \subseteq\left\{x: x^{\prime} \in B_{s, m},|x| \leq r_{s, m}\left(x^{\prime}\right)\right\},
$$

and using (4.6) with $r=r_{s, m}\left(x^{\prime}\right)$, we get

$$
\begin{aligned}
\gamma_{-1}\left(A_{s, m}\right) & \lesssim \int_{B_{s, m}} \int_{0}^{r_{s, m}\left(x^{\prime}\right)} r^{n-1} e^{r^{2}} d r d x^{\prime} \\
& \lesssim \int_{B_{s, m}} r_{s, m}\left(x^{\prime}\right)^{n-2} e^{r_{s, m}\left(x^{\prime}\right)^{2}} d x^{\prime} \\
& =\frac{1}{s} e^{-c 2^{2 m}} \int_{B_{s, m}} I_{m}\left(r_{s, m}\left(x^{\prime}\right), x^{\prime}\right) d x^{\prime} \\
& \leq \frac{1}{s} e^{-c 2^{2 m}} \int_{0}^{\infty} \rho^{2 n-2} \int_{\mathbf{S}^{n-1}} f\left(\rho y^{\prime}\right)\left(\int_{\left\{\left|y^{\prime}-x^{\prime}\right| \wedge\left|y^{\prime}+x^{\prime}\right|<2^{m+1} / \rho\right\}} d x^{\prime}\right) d y^{\prime} e^{\rho^{2}} d \rho \\
& \lesssim \frac{1}{s} e^{-c 2^{2 m}} \int_{0}^{\infty} \rho^{2 n-2}\left(\frac{2^{m}}{\rho}\right)^{n-1} \int_{\mathbf{S}^{n-1}} f\left(\rho y^{\prime}\right) d y^{\prime} e^{\rho^{2}} d \rho \\
& \approx \frac{1}{s} 2^{m(n-1)} e^{-c 2^{2 m}\|f\|_{L^{1}(\gamma-1)} .}
\end{aligned}
$$

In other words, $\left\|T_{m}\right\|_{L^{1}\left(\gamma_{-1}\right) \rightarrow L^{1, \infty}\left(\gamma_{-1}\right)} \lesssim 2^{m(n-1)} e^{-c 2^{2 m}}$. Because of (4.5) and (4.4), the proof is complete.

We conclude this section with another lemma, which will be involved in the study of the Riesz transforms of order 2.

Lemma 4.4. Let $\delta>0$. The operator with kernel

$$
K(x, y)=e^{-|x|^{2}+|y|^{2}} \frac{|x|^{\frac{n+1}{2}}}{\left|x-y_{x}\right|^{\frac{n-1}{2}}} e^{-\delta \frac{\left|y_{\perp}\right|^{2}|x|}{\left|x-y_{x}\right|}} \mathbf{1}_{\left\{|x|\left|x-y_{x}\right| \geq 1, \frac{1}{3}|x| \leq\left|y_{x}\right|<|x|\right\}}(x, y)
$$

is bounded from $L^{1}\left(\gamma_{-1}\right)$ to $L^{1, \infty}\left(\gamma_{-1}\right)$.

Proof. We begin with a series of observations that allow us to make some restrictions.

- If $n=1$, then $K(x, y) \leq e^{-|x|^{2}+|y|^{2}}|x|$, and the statement follows from Lemma 4.2. 
- If $\left|y_{\perp}\right|>|x| / 2$, then

$$
K(x, y) \lesssim \mathrm{e}^{-|x|^{2}+|y|^{2}} \frac{|x|^{\frac{n+1}{2}}}{\left|x-y_{x}\right|^{\frac{n-1}{2}}}\left(\frac{\left|x-y_{x}\right|}{\left|y_{\perp}\right|^{2}|x|}\right)^{\frac{n-1}{2}} \lesssim e^{-|x|^{2}+|y|^{2}}|x|^{2-n},
$$

and the statement follows again from Lemma 4.2.

- If $\left|y_{\perp}\right| \leq|x| / 2$ and $\theta>\pi / 6$, so that $\left|x-y_{x}\right| \approx|x|$, then

$$
K(x, y) \leq e^{-|x|^{2}+|y|^{2}}|x| e^{-c\left|y_{\perp}\right|^{2}} \mathbf{1}_{G \cap\left\{\frac{1}{3}|x| \leq|y| \leq 2|x|\right\}}(x, y),
$$

and we can apply Lemma 4.3.

- If $\frac{\left|y_{\perp}\right|^{2}|x|}{\left|x-y_{x}\right|}>\frac{1}{4}|x|^{2}$, then

$$
K(x, y) \lesssim e^{-|x|^{2}+|y|^{2}} \frac{|x|^{n}}{\left(|x|\left|x-y_{x}\right|\right)^{\frac{n-1}{2}}} e^{-c|x|^{2}} \mathbf{1}_{\left\{|x|\left|x-y_{x}\right| \geq 1\right\}}(x, y) \lesssim e^{-|x|^{2}+|y|^{2}}|x|^{-n},
$$

and the conclusion follows from Lemma 4.2.

- By means of a rotation, we can assume that $x$ is in the sector defined by $x_{1} /|x|>\sqrt{3} / 2$, that is, the angle between $x$ and the positive first coordinate axis is less than $\pi / 6$. Observe that if $\theta \leq \pi / 6$, then $y_{1} /|y|>1 / 2$.

Summing up all this, if $n \geq 2$ and we let

$$
\begin{gathered}
\Omega=\left\{(x, y) \in \mathbf{R}^{n} \times \mathbf{R}^{n}: \frac{x_{1}}{|x|}>\frac{\sqrt{3}}{2}, \frac{y_{1}}{|y|}>\frac{1}{2},|x|\left|x-y_{x}\right| \geq 1, \frac{1}{3}|x| \leq\left|y_{x}\right|<|x|,\right. \\
\left.\left|y_{\perp}\right| \leq|x|, \theta \in[0, \pi / 6], \frac{\left|y_{\perp}\right|^{2}|x|}{\left|x-y_{x}\right|} \leq \frac{1}{4}|x|^{2}\right\},
\end{gathered}
$$

then it is enough to prove the boundedness of the operator $\widetilde{T}$ whose kernel is

$$
\widetilde{K}(x, y)=e^{-|x|^{2}} \frac{|x|^{\frac{n+1}{2}}}{\left|x-y_{x}\right|^{\frac{n-1}{2}}} e^{-\frac{\left|y_{\perp}\right|^{2}|x|}{|x-y x|}} \mathbf{1}_{\Omega}(x, y),
$$

from $L^{1}$ (Leb) to $L^{1, \infty}\left(\gamma_{-1}\right)$. We observe that $\Omega \subset G$ and that $(x, y) \in \Omega$ implies $|y| \approx|x|$, so that $x$ and $y$ stay away from the origin.

For $(x, y) \in \Omega$, we have $|x|-\left|y_{x}\right|=\left|x-y_{x}\right|$, and it follows that

$$
\begin{aligned}
|x|-|y| & =\frac{|x|^{2}-|y|^{2}}{|x|+|y|}=\frac{\left(|x|-\left|y_{x}\right|\right)\left(|x|+\left|y_{x}\right|\right)-\left|y_{\perp}\right|^{2}}{|x|+|y|} \\
& \geq\left|x-y_{x}\right| \frac{|x|+\left|y_{x}\right|-|x| / 4}{|x|+|y|} \gtrsim\left|x-y_{x}\right| ;
\end{aligned}
$$

to estimate $\left|y_{\perp}\right|^{2}$ here, we used the last inequality in the definition of $\Omega$. In particular, $|y|<|x|$. Since also

$$
|x|-|y| \leq|x|-\left|y_{x}\right|=\left|x-y_{x}\right|
$$

we obtain

$$
|x|-|y| \approx\left|x-y_{x}\right| .
$$

Therefore, there exists $c>0$ such that

$\Omega \subseteq\left\{(x, y):|x|-|y| \geq \frac{c}{|x|}, \frac{1}{3}|x| \leq\left|y_{x}\right|,|y|<|x|, \theta \in[0, \pi / 6], \frac{\left|y_{\perp}\right|^{2}|x|}{\left|x-y_{x}\right|} \leq \frac{1}{4}|x|^{2}\right\}$. 
Let

$$
E_{0}=\left\{(x, y): \frac{\left|y_{\perp}\right|^{2}|x|}{\left|x-y_{x}\right|} \leq 1\right\} \quad \text { and } \quad E_{m}=\left\{(x, y): 2^{m-1}<\frac{\left|y_{\perp}\right|^{2}|x|}{\left|x-y_{x}\right|} \leq 2^{m}\right\},
$$

$m=1,2, \ldots$ Set also

$$
A_{0}=\{x:|x| \leq 1\} \quad \text { and } \quad A_{k}=\left\{x: 2^{k-1} \leq|x| \leq 2^{k}\right\}, \quad k=1,2, \ldots
$$

We define

$$
\widetilde{K}_{m, k}(x, y)=\widetilde{K}(x, y) \mathbf{1}_{E_{m}}(x, y) \mathbf{1}_{A_{k}}(x),
$$

so that

$$
\widetilde{K}=\sum_{m=0}^{\infty} \sum_{k=0}^{\infty} \widetilde{K}_{m, k}
$$

Observe that if $(x, y) \in \Omega$ and $x \in A_{k}$, then $y \in A_{k-2} \cup A_{k-1} \cup A_{k}$. For $(x, y) \in \Omega \cap E_{m}$ and $x \in A_{k}$, we make the transformation

$$
\xi_{1}=\frac{1}{2}|x|^{2}, \quad \xi_{j}=2^{-m / 2+2 k} \frac{x_{j}}{|x|}, \quad j=2, \ldots, n,
$$

and analogously

$$
\eta_{1}=\frac{1}{2}|y|^{2}, \quad \eta_{j}=2^{-m / 2+2 k} \frac{y_{j}}{|y|}, \quad j=2, \ldots, n .
$$

Going via the coordinates $\left(\xi_{1}, x_{2}, \ldots, x_{n}\right)$, one finds that the Jacobian of the transformation (4.8) is comparable to $x_{1} 2^{-\frac{m}{2}(n-1)} 2^{2 k(n-1)}|x|^{1-n}>0$, and similarly for (4.9). This leads to

$$
d x \approx 2^{\frac{m}{2}(n-1)} 2^{-n k} d \xi, \quad d y \approx 2^{\frac{m}{2}(n-1)} 2^{-n k} d \eta .
$$

Observe that $|x|-|y| \geq c /|x|$ implies

$$
\xi_{1}-\eta_{1}=\frac{1}{2}(|x|+|y|)(|x|-|y|) \gtrsim c,
$$

and that

$$
|x|-|y|=\sqrt{2}\left(\sqrt{\xi_{1}}-\sqrt{\eta_{1}}\right) \approx \frac{\xi_{1}-\eta_{1}}{\sqrt{\xi_{1}}} \approx \frac{\xi_{1}-\eta_{1}}{|x|} .
$$

Since $(x, y) \in \Omega \cap E_{m}$ and $x \in A_{k}$, we get for $j=2, \ldots, n$, using also (4.7), $\left|\xi_{j}-\eta_{j}\right| \lesssim 2^{-\frac{m}{2}+2 k} \sin \theta=2^{-\frac{m}{2}+2 k} \frac{\left|y_{\perp}\right|}{|y|} \lesssim 2^{k} \sqrt{\frac{|x|-|y|}{|x|}} \approx \sqrt{|x|(|x|-|y|)} \approx \sqrt{\xi_{1}-\eta_{1}}$. In other words, if we write $\xi^{\prime}$ for $\left(\xi_{2}, \ldots, \xi_{n}\right)$, and similarly for $\eta^{\prime}$, we obtain $\left|\xi^{\prime}-\eta^{\prime}\right| \lesssim$ $\sqrt{\xi_{1}-\eta_{1}}$.

These transformations lead us to the operator

$$
\mathcal{T}_{m, k} \varphi(\xi)=\int_{\mathbf{R}^{n}} \mathcal{K}_{m, k}(\xi, \eta) \varphi(\eta) d \eta
$$

where

$$
\begin{aligned}
& \mathcal{K}_{m, k}(\xi, \eta) \\
& \left.=\mathbf{1}_{\left[2^{2 k-3}, 2^{2 k-1}\right]}\left(\xi_{1}\right) e^{-2^{m-1}} 2^{\frac{m}{2}(n-1)} e^{-2 \xi_{1}}\left(\xi_{1}-\eta_{1}\right)^{\frac{1-n}{2}} \mathbf{1}_{\left\{\left|\xi^{\prime}-\eta^{\prime}\right| \lesssim \sqrt{\xi_{1}-\eta_{1}}\right.}, \frac{\xi_{1}}{9}<\eta_{1}<\xi_{1}-c\right\} \\
&
\end{aligned}
$$

Indeed, the reader may verify that the operator with kernel $\widetilde{K}_{m, k}$ is bounded from $L^{1}\left(\right.$ Leb) to $L^{1, \infty}\left(\gamma_{-1}\right)$ if $\mathcal{T}_{m, k}$ is bounded from $L^{1}(d \eta)$ to $L^{1, \infty}\left(e^{2 \xi_{1}} d \xi\right)$, with uniform 
control in $m$ and $k$ of the quotients between the operator quasi-norms. We thus verify that $\mathcal{T}_{m, k}$ has this boundedness property, uniformly in $m$ and $k$.

By [7, Proposition 8], $\mathcal{T}_{m, k}$ is bounded from $L^{1}(d \eta)$ to $L^{1, \infty}\left(e^{2 \xi_{1}} d \xi\right)$, with norm $\lesssim e^{-2^{m-1}} 2^{\frac{n-1}{2} m}$ uniformly in $k$. A similar estimate thus holds for the norm from $L^{1}\left(\right.$ Leb) to $L^{1, \infty}\left(\gamma_{-1}\right)$ of the operator whose kernel is $\widetilde{K}_{m, k}$.

Since $y \in A_{k-2} \cup A_{k-1} \cup A_{k}$ if $x \in A_{k}$ and $(x, y) \in \Omega$, we can sum over $k$ and conclude that the operator with kernel $\widetilde{K} \mathbf{1}_{E_{m}}$ is bounded from $L^{1}\left(\right.$ Leb) to $L^{1, \infty}\left(\gamma_{-1}\right)$ with norm controlled by $e^{-2^{m-1}} 2^{\frac{n-1}{2} m}$. Finally, the proof is completed with a summation in $m$ and use of (4.4).

\section{The global part of the Riesz trasforms}

Proposition 5.1. If $|\alpha| \in\{1,2\}$, then $\mathscr{R}_{\alpha, \text { glob }}$ is bounded from $L^{1}\left(\gamma_{-1}\right)$ to $L^{1, \infty}\left(\gamma_{-1}\right)$.

Proof. Let $(x, y) \in G$. By $(2.3)$,

$$
\begin{aligned}
& \left|K_{\mathscr{R}_{\alpha}}(x, y)\right| \leq c(|\alpha|) e^{-|x|^{2}+|y|^{2}} \int_{0}^{1} \frac{r^{n-1}(-\log r)^{\frac{|\alpha|}{2}-1}}{\left(1-r^{2}\right)^{\frac{n+|\alpha|}{2}}}\left|H_{\alpha}\left(\frac{x-r y}{\sqrt{1-r^{2}}}\right)\right| e^{-\frac{|r x-y|^{2}}{1-r^{2}}} d r \\
& \lesssim e^{-|x|^{2}+|y|^{2}} \sum_{a=0}^{|\alpha|}\left(\int_{0}^{1 / 2} r^{n-1}|x-r y|^{a} e^{-|r x-y|^{2}} d r+\int_{1 / 2}^{1} \frac{|x-r y|^{a}}{(1-r)^{\frac{n+2+a}{2}}} e^{-\frac{|r x-y|^{2}}{1-r^{2}}} d r\right) \\
& =e^{-|x|^{2}+|y|^{2}} \sum_{a=0}^{|\alpha|}\left(K_{1}^{a}(x, y)+K_{2}^{a}(x, y)\right),
\end{aligned}
$$

say. We separate the analyses of $K_{1}^{a}$ and $K_{2}^{a}$.

Step 1. We prove that $e^{-|x|^{2}+|y|^{2}} K_{1}^{a}(x, y)$ is the kernel of an operator of weak type $(1,1)$. Since

$$
K_{1}^{a}(x, y) \lesssim \int_{0}^{1 / 2} r^{n-1}\left(|x|^{a}+|y|^{a}\right) e^{-|r x-y|^{2}} d r
$$

we consider two cases, depending on the values of the quotient $|y| /|x|$.

First, suppose $|y| /|x| \geq 2$. Then $|r x-y|>3|y| / 4$ for $0<r<1 / 2$, so that

$$
K_{1}^{a}(x, y) \lesssim \int_{0}^{1 / 2} r^{n-1}|y|^{a} e^{-c|y|^{2}} d r \lesssim|y|^{a} e^{-c|y|^{2}} \lesssim|y|^{1-n} \lesssim|x|^{1-n},
$$

and Lemma 4.2 applies.

We now consider the case $|y| /|x|<2$. Then

$$
\begin{aligned}
& K_{1}^{a}(x, y) \lesssim e^{-\left|y_{\perp}\right|^{2}} \int_{0}^{1 / 2}\left[\left|r_{0}\right|^{n-1}+\left|r-r_{0}\right|^{n-1}\right]|x|^{a} e^{-\left|r-r_{0}\right|^{2}|x|^{2}} d r \\
& \lesssim e^{-\left|y_{\perp}\right|^{2}}\left[\left(\frac{|y|}{|x|}\right)^{n-1}|x|^{a} \int_{0}^{1 / 2} e^{-\left|r-r_{0}\right|^{2}|x|^{2}} d r+|x|^{a} \int_{0}^{1 / 2}\left|r-r_{0}\right|^{n-1} e^{-\left|r-r_{0}\right|^{2}|x|^{2}} d r\right] \\
& \lesssim e^{-\left|y_{\perp}\right|^{2}}|x|^{a-1}\left(\frac{|y|}{|x|}\right)^{n-1}+|x|^{a-n} .
\end{aligned}
$$

Since $|x| \geq c$ for $|x|>|y| / 2$ and $(x, y) \in G$, and also $a \leq 2$, we can apply Lemmata 4.3 and 4.2 and complete Step 1. 
Step 2. We prove that $e^{-|x|^{2}+|y|^{2}} K_{2}^{a}(x, y)$ is the kernel of an operator of weak type $(1,1)$.

By (4.1),

$$
\begin{gathered}
K_{2}^{a}(x, y)=\int_{1 / 2}^{1} \frac{|x-r y|^{a}}{(1-r)^{\frac{n+a+2}{2}}} e^{-c \frac{\left(r-r_{0}\right)^{2}|x|^{2}+\left|y_{\perp}\right|^{2}}{1-r}} d r \\
=\int_{1 / 2}^{1}\left(\mathbf{1}_{\left\{r_{0} \leq 1 / 3\right\}}+\mathbf{1}_{\left\{r_{0} \geq 2\right\}}+\mathbf{1}_{\left\{1 / 3<r_{0}<2\right\}}\right)(x, y) \frac{|x-r y|^{a}}{(1-r)^{\frac{n+a+2}{2}}} e^{-c \frac{\left(r-r_{0}\right)^{2}|x|^{2}+\left|y_{\perp}\right|^{2}}{1-r}} d r \\
=: K_{2,1}^{a}(x, y)+K_{2,2}^{a}(x, y)+K_{2,3}^{a}(x, y) .
\end{gathered}
$$

We prove separately the weak type $(1,1)$ of the operators associated to the kernels $e^{-|x|^{2}+|y|^{2}} K_{2, i}^{a}(x, y), i=1,2,3$.

2.1. If $r_{0} \leq 1 / 3$ and $1 / 2 \leq r \leq 1$, then

$$
\left|r-r_{0}\right| \approx 1+\left|r_{0}\right| \text {. }
$$

Moreover,

$$
|x-r y| \lesssim\left(1+\left|r_{0}\right|\right)|x|+\left|y_{\perp}\right| \text {. }
$$

Therefore, with the change of variables $\frac{\left(1+\left|r_{0}\right|\right)^{2}|x|^{2}+\left|y_{\perp}\right|^{2}}{1-r}=s$,

$$
\begin{aligned}
K_{2,1}^{a}(x, y) & \lesssim\left[\left(1+\left|r_{0}\right|\right)|x|+\left|y_{\perp}\right|\right]^{a} \int_{1 / 2}^{1} \frac{1}{(1-r)^{\frac{n+a+2}{2}}} e^{-c \frac{\left(1+\left|r_{0}\right|\right)^{2}|x|^{2}+\left|y_{\perp}\right|^{2}}{1-r}} d r \\
& \lesssim\left[\left(1+\left|r_{0}\right|\right)^{2}|x|^{2}+\left|y_{\perp}\right|^{2}\right]^{-\frac{n}{2}} \\
& \lesssim|x|^{-n} .
\end{aligned}
$$

Thus, the operator with kernel $e^{-|x|^{2}+|y|^{2}} K_{2,1}^{a}(x, y)$ is of weak type $(1,1)$ by Lemma 4.2 .

2.2. If $r_{0} \geq 2$, then (5.1) and (5.2) remain valid if $1 / 2 \leq r \leq 1$ and we can argue as in the preceding case.

2.3. We split the integral defining $K_{2,3}^{a}$ in a way that depends on the value of $r_{0}$. Let

$$
\begin{aligned}
& I_{1}=\left[\frac{1}{2}, 1\right) \cap\left\{r: 1-r>\frac{3}{2}\left|1-r_{0}\right|\right\}, \\
& I_{2}=\left[\frac{1}{2}, 1\right) \cap\left\{r: 1-r \leq \frac{1}{2}\left(1-r_{0}\right) \vee \frac{3}{2}\left(r_{0}-1\right)\right\}, \\
& I_{3}=\left[\frac{1}{2}, 1\right) \cap\left\{r:\left|r-r_{0}\right|<\frac{1}{2}\left(1-r_{0}\right)\right\},
\end{aligned}
$$

and for $j=1,2,3$ define

$$
K_{2,3, j}^{a}(x, y)=\mathbf{1}_{\left\{1 / 3<r_{0}<2\right\}}(x, y) \int_{I_{j}} \frac{|x-r y|^{a}}{(1-r)^{\frac{n+a+2}{2}}} e^{-c \frac{\left(r-r_{0}\right)^{2}|x|^{2}+\left|y_{\perp}\right|^{2}}{1-r}} d r .
$$

Thus, $K_{2,3}^{a}=K_{2,3,1}^{a}+K_{2,3,2}^{a}+K_{2,3,3}^{a}$. It will be useful to observe that, since $r_{0} \approx 1$, or equivalently $\left|y_{x}\right| \approx|x|$, and $\theta<\pi / 6$, one has

$$
\left|y_{\perp}\right|^{-n} \lesssim(|x| \sin \theta)^{-n}
$$

We first consider $K_{2,3,1}^{a}$. Since

$$
1-r-\left|r_{0}-1\right| \leq r_{0}-r \leq 1-r+\left|r_{0}-1\right|,
$$


the condition $1-r>\frac{3}{2}\left|1-r_{0}\right|$ implies $\left|r-r_{0}\right| \approx 1-r$. Thus,

$$
|x|^{2} \frac{\left(r-r_{0}\right)^{2}}{1-r} \approx|x|^{2}(1-r)
$$

and (4.3) implies

$$
|x-r y| \leq\left|1-r_{0}\right||x|+(1-r)\left|y_{x}\right|+\left|y_{\perp}\right| \lesssim(1-r)|x|+\left|y_{\perp}\right| .
$$

We then get

$$
\begin{aligned}
K_{2,3,1}^{a}(x, y) \lesssim & \int_{1-r>3\left|1-r_{0}\right| / 2}(1-r)^{-\frac{n+2}{2}}|x|^{a}(1-r)^{\frac{a}{2}} e^{-c|x|^{2}(1-r)} e^{-c \frac{|y \perp|^{2}}{1-r}} d r \\
& +\int_{1-r>3\left|1-r_{0}\right| / 2}(1-r)^{-\frac{n+2}{2}} \frac{\left|y_{\perp}\right|^{a}}{(1-r)^{\frac{a}{2}}} e^{-c|x|^{2}(1-r)} e^{-c \frac{\left|y_{\perp}\right|^{2}}{1-r}} d r \\
\lesssim & \int_{1-r>3\left|1-r_{0}\right| / 2}(1-r)^{-\frac{n+2}{2}} e^{-c|x|^{2}(1-r)} e^{-c \frac{\mid y \perp}{1-r}} d r
\end{aligned}
$$

By the change of variables $\left|y_{\perp}\right|^{2} /(1-r)=s$, one obtains

We also have

$$
K_{2,3,1}^{a}(x, y) \lesssim \frac{1}{\left|y_{\perp}\right|^{n}}
$$

$$
K_{2,3,1}^{a}(x, y) \lesssim \int_{1-r>3\left|1-r_{0}\right| / 2}(1-r)^{-\frac{n+2}{2}} d r \lesssim\left|1-r_{0}\right|^{-\frac{n}{2}} \approx|x|^{\frac{n}{2}}\left|x-y_{x}\right|^{-\frac{n}{2}},
$$

in view of (4.2). Since $(1+|x|)\left|x-y_{x}\right| \gtrsim 1$ if $\left|x-y_{x}\right|>\left|y_{\perp}\right|$, while $(1+|x|)\left|y_{\perp}\right| \gtrsim 1$ if $\left|x-y_{x}\right| \leq\left|y_{\perp}\right|($ recall $(x, y) \in G)$, we obtain

$$
K_{2,3,1}^{a}(x, y) \lesssim(1+|x|)^{n} .
$$

In other words,

$$
K_{2,3,1}^{a}(x, y) \lesssim(1+|x|)^{n} \wedge(|x| \sin \theta)^{-n},
$$

and so the operator with kernel $e^{-|x|^{2}+|y|^{2}} K_{2,3,1}^{a}(x, y)$ is of weak type $(1,1)$ by Lemma 4.1.

We now consider $K_{2,3,2}^{a}$. Here $\left|1-r_{0}\right| \approx\left|r-r_{0}\right|$, since

$$
\left|r-r_{0}\right|=\left|1-r_{0}-(1-r)\right| \leq\left|1-r_{0}\right|+(1-r) \lesssim\left|1-r_{0}\right| \text {. }
$$

If $r_{0}<1$, then $1-r<\frac{1}{2}\left(1-r_{0}\right)$ and hence

$$
\left|r-r_{0}\right|=\left|1-r_{0}-(1-r)\right| \geq\left|1-r_{0}\right|-|1-r| \geq \frac{1}{2}\left|1-r_{0}\right| .
$$

If $r_{0}>1$, then

$$
\left|r-r_{0}\right|=r_{0}-r>r_{0}-1=\left|1-r_{0}\right| .
$$

In both cases, by (4.3),

$$
|x-r y| \lesssim\left|x-y_{x}\right|+\left|y_{\perp}\right|
$$

since $1-r \lesssim\left|1-r_{0}\right|,\left|y_{x}\right| \approx|x|$ and by (4.2). Thus

$$
K_{2,3,2}^{a}(x, y) \lesssim\left(\left|x-y_{x}\right|+\left|y_{\perp}\right|\right)^{a} \int_{0}^{1}(1-r)^{-\frac{n+a+2}{2}} e^{-c \frac{\left|x-y_{x}\right|^{2}+\left|y_{\perp}\right|^{2}}{1-r}} d r .
$$

After the change of variables $\frac{\left|x-y_{x}\right|^{2}+\left|y_{\perp}\right|^{2}}{1-r}=s$, we obtain

$$
K_{2,3,2}^{a}(x, y) \lesssim\left(\left|x-y_{x}\right|+\left|y_{\perp}\right|\right)^{-n} \leq \frac{1}{|x-y|^{n}} \wedge \frac{1}{\left|y_{\perp}\right|^{n}} \lesssim(1+|x|)^{n} \wedge \frac{1}{(|x| \sin \theta)^{n}}
$$

Lemma 4.1 now ends the case of $K_{2,3,2}^{a}$. 
It remains to consider $K_{2,3,3}^{a}$. Observe first that $K_{2,3,3}^{a}=K_{2,3,3}^{a} \mathbf{1}_{\left\{1 / 3<r_{0}<1\right\}}$. Then, the conditions $1 / 3<r_{0}<1$ and $\left|r-r_{0}\right|<\frac{1}{2}\left(1-r_{0}\right)$ imply $1-r \approx 1-r_{0}$. Moreover, by (4.3), the fact that $\left|y_{x}\right| \approx|x|$ and (4.2) we get

$$
|x-r y| \lesssim\left(1-r_{0}\right)|x|+\left|y_{\perp}\right| \text {. }
$$

Therefore

$$
\begin{aligned}
& K_{2,3,3}^{a}(x, y) \lesssim\left(1-r_{0}\right)^{-\frac{n+a+2}{2}}\left[\left(1-r_{0}\right)|x|+\left|y_{\perp}\right|\right]^{a} \int_{\left|r-r_{0}\right|<\frac{1-r_{0}}{2}} e^{-c|x|^{2} \frac{\left(r-r_{0}\right)^{2}}{1-r_{0}}-c\left|y_{\perp}\right|^{2} \frac{1}{1-r_{0}}} d r \\
& \lesssim\left[\left(1-r_{0}\right)^{-\frac{n-a+2}{2}}|x|^{a}+\left(1-r_{0}\right)^{-\frac{n+a+2}{2}}\left|y_{\perp}\right|^{a}\right] e^{-c \frac{\left|y_{\perp}\right|^{2}}{1-r_{0}}} \int_{\left|r-r_{0}\right|<\frac{1-r_{0}}{2}} e^{-c|x|^{2} \frac{\left(r-r_{0}\right)^{2}}{1-r_{0}}} d r .
\end{aligned}
$$

Observe now that

$$
\int_{\left|r-r_{0}\right|<\frac{1-r_{0}}{2}} e^{-c|x|^{2} \frac{\left(r-r_{0}\right)^{2}}{1-r_{0}}} d r \lesssim \frac{\sqrt{1-r_{0}}}{|x|} \wedge\left(1-r_{0}\right) .
$$

Therefore,

$$
K_{2,3,3}^{a}(x, y) \lesssim A^{a}(x, y)+B^{a}(x, y)
$$

where

$$
A^{a}(x, y)=\left(1-r_{0}\right)^{-\frac{n-a+1}{2}}|x|^{a-1} e^{-\frac{|y \perp|^{2}}{1-r_{0}}}\left(1 \wedge|x| \sqrt{1-r_{0}}\right) \mathbf{1}_{\left\{\frac{1}{3}<r_{0}<1\right\}}(x, y)
$$

and

$$
B^{a}(x, y)=\left(1-r_{0}\right)^{-\frac{n+a+2}{2}}\left|y_{\perp}\right|^{a} e^{-c \frac{\left|y_{\perp}\right|^{2}}{1-r_{0}}}\left(\frac{\sqrt{1-r_{0}}}{|x|} \wedge\left(1-r_{0}\right)\right) .
$$

Observe that

$$
\begin{aligned}
B^{a}(x, y) & \lesssim\left(1-r_{0}\right)^{-\frac{n+a+2}{2}}\left|y_{\perp}\right|^{a} \frac{\sqrt{1-r_{0}}}{|x|}\left(\frac{\sqrt{1-r_{0}}}{\left|y_{\perp}\right|}\right)^{a} e^{-\frac{c}{2} \frac{\left|y_{\perp}\right|^{2}}{1-r_{0}}} \\
& \lesssim\left(1-r_{0}\right)^{-\frac{n+1}{2}} \frac{1}{|x|} e^{-\frac{c}{2} \frac{\left|y_{\perp}\right|^{2}}{1-r_{0}}} .
\end{aligned}
$$

Now, if $\left|y_{x}-x\right| \geq\left|y_{\perp}\right|$, then $\left|y_{x}-x\right| \gtrsim(1+|x|)^{-1}$ since $(x, y) \in G$, and (4.2) implies

$$
B^{a}(x, y) \lesssim\left(1-r_{0}\right)^{-\frac{n+1}{2}} \frac{1}{|x|} \lesssim(1+|x|)^{n} .
$$

But if $\left|y_{x}-x\right|<\left|y_{\perp}\right|$, then $\left|y_{\perp}\right| \gtrsim(1+|x|)^{-1}$ since $(x, y) \in G$, and again by (4.2)

$$
B^{a}(x, y) \lesssim\left(1-r_{0}\right)^{-\frac{n+1}{2}}\left(\frac{1-r_{0}}{\left|y_{\perp}\right|^{2}}\right)^{\frac{n+1}{2}} \frac{1}{|x|} \lesssim(1+|x|)^{n} .
$$

On the other hand, by the definition of $B^{a}(x, y)$

$$
B^{a}(x, y) \lesssim\left(1-r_{0}\right)^{-\frac{n+a+2}{2}}\left|y_{\perp}\right|^{a}\left(\frac{1-r_{0}}{\left|y_{\perp}\right|^{2}}\right)^{\frac{n+a}{2}}\left(1-r_{0}\right) \lesssim(|x| \sin \theta)^{-n}
$$

Lemma 4.1 applies, and the operator with kernel $e^{-|x|^{2}+|y|^{2}} B^{a}(x, y)$ is of weak type $(1,1)$.

We now estimate $A^{a}$. Arguing as in the two cases for $B^{a}$, one can show that

$$
A^{a}(x, y) \lesssim(1+|x|)^{n} .
$$


Now, by means of (4.2) we rewrite $A^{a}$ as

$$
A^{a}(x, y)=\frac{|x|^{\frac{n+a-1}{2}}}{\left|x-y_{x}\right|^{\frac{n-a+1}{2}}} e^{-c \frac{\left|y_{\perp}\right|^{2}|x|}{|x-y x|}}\left(1 \wedge \sqrt{|x|\left|x-y_{x}\right|}\right) \mathbf{1}_{\left\{\frac{1}{3}<r_{0}<1\right\}}(x, y) .
$$

If $a=0$, then

$$
A^{0}(x, y) \lesssim \frac{|x|^{\frac{n-1}{2}}}{\left|x-y_{x}\right|^{\frac{n+1}{2}}}\left(\frac{\left|x-y_{x}\right|}{|x|\left|y_{\perp}\right|^{2}}\right)^{n / 2} \sqrt{|x|\left|x-y_{x}\right|} \lesssim(|x| \sin \theta)^{-n} .
$$

If $a=1$, then

$$
A^{1}(x, y) \lesssim \frac{|x|^{\frac{n}{2}}}{\left|x-y_{x}\right|^{\frac{n}{2}}}\left(\frac{\left|x-y_{x}\right|}{|x|\left|y_{\perp}\right|^{2}}\right)^{n / 2} \lesssim(|x| \sin \theta)^{-n} .
$$

Because of (5.4), (5.5) and (5.6), the operators with kernels $e^{-|x|^{2}+|y|^{2}} A^{0}(x, y)$ and $e^{-|x|^{2}+|y|^{2}} A^{1}(x, y)$ are of weak type $(1,1)$ by Lemma 4.1 .

If $a=2$, then

$$
A^{2}(x, y)=\frac{|x|^{\frac{n+1}{2}}}{\left|x-y_{x}\right|^{\frac{n-1}{2}}} e^{-c \frac{\left|y_{\perp}\right|^{2}|x|}{|x-y x|}}\left(1 \wedge \sqrt{|x|\left|x-y_{x}\right|}\right) \mathbf{1}_{\left\{\frac{1}{3}<r_{0}<1\right\}}(x, y) .
$$

If $|x|\left|x-y_{x}\right| \leq 1$, then

$$
A^{2}(x, y) \lesssim \frac{|x|^{\frac{n+1}{2}}}{\left|x-y_{x}\right|^{\frac{n-1}{2}}}\left(\frac{\left|x-y_{x}\right|}{\left|y_{\perp}\right|^{2}|x|}\right)^{n / 2} \sqrt{|x|\left|x-y_{x}\right|} \lesssim(|x| \sin \theta)^{-n} .
$$

By this and (5.4), the operator whose kernel is $e^{-|x|^{2}+|y|^{2}} A^{2}(x, y) \mathbf{1}_{\left\{|x|\left|x-y_{x}\right| \leq 1\right\}}$ is of weak type $(1,1)$ because of Lemma 4.1. The operator whose kernel is $e^{-|x|^{2}+|y|^{2}}$ $\cdot A^{2}(x, y) \mathbf{1}_{\left\{|x|\left|x-y_{x}\right| \geq 1\right\}}$ is also of weak type $(1,1)$, by Lemma 4.4. This concludes the proof of Step 2 and that of the theorem.

\section{Unboundedness of the Riesz transforms of order at least three}

In this section, we complete the proof of Theorem 1.1. The proof of the following proposition is inspired by that of [6, Section 5], which we adapt to the current setting.

Proposition 6.1. If $|\alpha| \geq 3$, then $\mathscr{R}_{\alpha}$ is unbounded from $L^{1}\left(\gamma_{-1}\right)$ to $L^{1, \infty}\left(\gamma_{-1}\right)$.

Proof. Let $\eta>0$ be large, and write $z$ for the point $(\eta, \ldots, \eta) \in \mathbf{R}^{n}$. For every $x \in \mathbf{R}^{n}$, denote by $x_{z}$ the component of $x$ which is parallel to $z$, and by $x_{\perp}=x-x_{z}$ the component orthogonal to $z$.

Define the tube

$$
J(z)=\left\{x \in \mathbf{R}^{n}:\left|x_{\perp}\right|<1, \frac{4}{3}|z|<\left|x_{z}\right|<\frac{3}{2}|z|\right\} .
$$

We claim that if $\eta$ is sufficiently large, then for every $r \in(0,1), y \in B(z, 1)$ and $x \in J(z)$ one has

Indeed, for these $r, y, x$,

$$
\frac{x_{i}-r y_{i}}{\sqrt{1-r^{2}}} \gtrsim|z|, \quad i=1, \ldots, n
$$

$$
\left|(x-r y)_{i}-\left(x_{z}-r z\right)_{i}\right| \leq\left|x-r y-x_{z}+r z\right| \leq 2 \quad \text { and } \quad x_{z}-r z=c(r) z,
$$

where $c(r)>1 / 3$ for $r \in(0,1)$. The claim follows, and with $\eta$ large we also conclude that

$$
H_{\alpha}\left(\frac{x-r y}{\sqrt{1-r^{2}}}\right) \gtrsim|z|^{|\alpha|}
$$


Moreover, if $x \in J(z), y \in B(z, 1)$ and $1 / 4<r<3 / 4$,

$$
e^{-\frac{|r x-y|^{2}}{1-r^{2}}} \geq e^{-2 \frac{|r x-z|^{2}}{1-r^{2}}-2 \frac{|z-y|^{2}}{1-r^{2}}} \gtrsim e^{-c|r x-z|^{2}}=e^{-c r^{2}\left|x_{\perp}\right|^{2}-c\left|r x_{z}-z\right|^{2}} \gtrsim e^{-\left.c|r| x_{z}|-| z\right|^{2}} .
$$

Thus, if $\eta$ is sufficiently large, $x \in J(z)$ and $y \in B(z, 1)$, then (recall (2.3))

$$
(-1)^{|\alpha|} K_{\mathscr{R}_{\alpha}}(x, y) \gtrsim|z|^{|\alpha|} e^{-|x|^{2}+|y|^{2}} \int_{1 / 4}^{3 / 4} e^{-c|r| x z|-| z||^{2}} d r \gtrsim|z|^{|\alpha|-1} e^{-|x|^{2}+|y|^{2}} .
$$

Take now a function $f \geq 0$ supported in the ball $B(z, 1)$ and such that $\|f\|_{L^{1}(\gamma-1)}=1$. By (6.1), for $x \in J(z)$ and $\eta$ sufficiently large, we have

$$
\left|\mathscr{R}_{\alpha} f(x)\right| \gtrsim e^{-|x|^{2}}|z|^{|\alpha|-1} \gtrsim e^{-\left(\frac{3}{2}|z|\right)^{2}}|z|^{|\alpha|-1} .
$$

Since

we conclude that

$$
\gamma_{-1}(J(z)) \gtrsim e^{\left(\frac{3}{2}|z|\right)^{2}}|z|^{-1}
$$

$$
\sup _{s>0} s \gamma_{-1}\left\{x:\left|\mathscr{R}_{\alpha} f(x)\right|>s\right\} \gtrsim e^{-\left(\frac{3}{2}|z|\right)^{2}}|z|^{|\alpha|-1} \gamma_{-1}(J(z)) \gtrsim \eta^{|\alpha|-2},
$$

from which the proposition follows.

\section{References}

[1] Auscher, P., T. Coulhon, X. T. Duong, and S. Hofmann: Riesz transform on manifolds and heat kernel regularity. - Ann. Sci. École Norm. Sup. (4) 37:6, 2004, 911-957.

[2] BAKRY, D.: Étude des transformations de Riesz dans les variétés riemanniennes à courbure de Ricci minorée. - Séminaire de Probabilités, XXI, 137-172.

[3] Bruno, T.: Endpoint results for the Riesz transform of the Ornstein-Uhlenbeck operator. J. Fourier Anal. Appl. 25:4, 2019, 1609-1631.

[4] Bruno, T.: Singular integrals and Hardy type spaces for the inverse Gauss measure. - J. Geom. Anal. (to appear).

[5] Coulhon, T., and X. T. Duong: Riesz transforms for $1 \leq p \leq 2$. - Trans. Amer. Math. Soc. 351:3, 1999, 1151-1169.

[6] García-Cuerva, J., G. Mauceri, P. Sjögren, and J. L. Torrea: Higher-order Riesz operators for the Ornstein-Uhlenbeck semigroup. - Potential Anal. 10:4, 1999, 379-407.

[7] Li, H.-Q., and P. SJöGREN: Sharp endpoint estimates for some operators associated with the Laplacian with drift in Euclidean space. - Canadian J. Math. (to appear).

[8] PÉrez, S., and F. Soria: Operators associated with the Ornstein-Uhlenbeck semigroup. - J. London Math. Soc. (2) 61:3, 2000, 857-871.

[9] Salogni, F.: Harmonic Bergman spaces, Hardy-type spaces and harmonic analysis of a symmetric diffusion semigroup on $\mathbf{R}^{n}$. - PhD Thesis, Università degli Studi di Milano-Bicocca, https://boa.unimib.it/retrieve/handle/10281/41814/62217/phd_unimib_058626.pdf, 2013.

[10] Stein, E., and N. Weiss: On the convergence of Poisson integrals. - Trans. Amer. Math. Soc. $140,1969,35-54$.

[11] SzegÖ, G.: Orthogonal polynomials. Fourth edition. - Colloquium Publications, Vol. XXIII, Amer. Math. Soc., Providence, R.I., 1975. 